\title{
ON THE SOLVABILITY OF $\mathbb{Z}_{3}$-GRADED NOVIKOV ALGEBRAS
}

\author{
Viktor Zhelyabin ${ }^{1}$ \\ and \\ Ualbai Umirbaev ${ }^{2}$
}

\begin{abstract}
Symmetries of algebraic systems are called automorphisms. An algebra, over a good field, admits an automorphism of finite order $n$ if and only if it admits a $\mathbb{Z}_{n}$-grading. Let $N=N_{0} \oplus N_{1} \oplus N_{2}$ be a $\mathbb{Z}_{3}$-graded Novikov algebra. The main goal of the paper is to prove that over a field of characteristic not equal to 3 the algebra $N$ is solvable if $N_{0}$ is solvable. We also show that a $\mathbb{Z}_{2}$-graded Novikov algebra $N=N_{0} \oplus N_{1}$ over a field of characteristic not equal to 2 is solvable if $N_{0}$ is solvable. This implies that for every $n$ of the form $n=2^{k} 3^{l}$, any $\mathbb{Z}_{n}$-graded Novikov algebra $N$ over a field of characteristic not equal to 2,3 is solvable if $N_{0}$ is solvable.
\end{abstract}

Key words: Novikov algebra, graded algebra, solvability, regular automorphism, the ring of invariants

\section{INTRODUCTION}

One of the features of Hamiltonian operators is their connection with certain algebraic structures [1-7]. In 1976 I.M. Gel'fand and L.A. Dikii [1] introduced formal variational calculus and found some interesting Poisson structures when studying Hamiltonian systems related to some nonlinear partial differential equations such as the Korteweg-de Vries equations. A little later, I.M. Gel'fand and I.Ya. Dorfman [2] found more connections between Hamiltonian operators and some algebraic structures. In 1983-85 B.A. Dubrovin, A.A. Balanskii and S.P. Novikov [3-5] studied similar Poisson structures from another point of view. One of the algebraic structures in $[2,5]$, introduced in connection with Poisson brackets of hydrodynamic type, was called a Novikov algebra by J.M. Osborn [8-10].

We recall some important results on the solvabilty and nilpotency of Novikov algebras. In 1987 E.I. Zelmanov [11] proved that if $N$ is a finite dimensional right nilpotent Novikov algebra, then $N^{2}$ is nilpotent. In 2001 V.T. Filippov [12] proved that any left-nil Novikov algebra of bounded index over a field of characteristic zero is nilpotent. Recently I. Shestakov and Z. Zhang [13] showed that a Novikov algebra is solvable if and only if it is right nilpotent.

Symmetries of algebraic systems are called automorphisms. The most famous example of symmetry in algebra is related to the action of the symmetric group $S_{n}$ on a polynomial algebra $F\left[x_{1}, \ldots, x_{n}\right]$ in the variables $x_{1}, \ldots, x_{n}$. The algebra of invariants of this action is a polynomial algebra generated by elementary symmetric polynomials.

\footnotetext{
${ }^{1}$ Institute of Mathematics of the SB of RAS, Novosibirsk, 630090, Russia, e-mail: vicnic@math.nsc.ru

${ }^{2}$ Wayne State University, Detroit, MI 48202, USA, and Institute of Mathematics and Modeling, Almaty, Kazakhstan, e-mail: umirbaev@wayne.edu
} 
Let $R$ be an algebra over a field $F$. For any automorphism $\phi$ of $R$ the set of fixed elements $R^{\phi}=\{x \in R \mid \phi(x)=x\}$ is a subalgebra of $R$, and is called the subalgebra of invariants of $\phi$. An automorphism $\phi$ is called regular if $R^{\phi}=0$. For any group $G$ of automorphisms of $R$ the subalgebra of invariants $R^{G}=\{x \in R \mid \phi(x)=x$ for all $\phi \in G\}$ is defined similarly.

In 1957 G. Higman [14] published a classical result on Lie algebras which says that if a Lie algebra $L$ has a regular automorphism $\phi$ of prime order $p$, then $L$ is nilpotent. It was also shown that the index of nilpotency $h(p)$ of $L$ depends only on $p$. An explicit estimation of the function $h(p)$ was found by A.I. Kostrikin and V.A. Kreknin [15] in 1963. A little later, V.A. Kreknin proved [16] that a finite dimensional Lie algebra with a regular automorphism of an arbitrary finite order is solvable. In $2005 \mathrm{~N}$. Yu. Makarenko [17] proved that if a Lie algebra $L$ admits an automorphism of a prime order $p$ with a finite-dimensional fixed subalgebra of dimension $t$, then $L$ has a nilpotent ideal of finite codimension with the index of nilpotency bounded in terms of $p$ and the codimension bounded in terms of $t$ and $p$.

In 1973 G. Bergman and I. Isaacs [18] published a classical result on the actions of finite groups on associative algebras. Let $G$ be a finite group of automorphisms of an associative algebra $R$ and suppose that $R$ has no $|G|$-torsion. If the subalgebra of invariants $R^{G}$ is nilpotent, then the Bergman-Isaacs Theorem [18] states that $R$ is also nilpotent. Since then a very large number of papers have been devoted to the study of automorphisms of associative rings. The central problem of these studies was to identify the properties of rings that can be transformed from the ring of invariants to the whole ring. In $1974 \mathrm{~V}$. $\mathrm{K}$. Kharchenko [19] proved if $R^{G}$ is a PI-ring, then $R$ is a PI-ring under the conditions of the Bergman-Isaacs Theorem.

The Bergman-Isaacs Theorem was partially generalized by W.S. Martindale and S. Montgomery [20] in 1977 to the case of a finite group of Jordan automorphisms, that is, a finite group of automorphisms of the adjoint Jordan algebra $R^{(+)}$.

An analogue of Kharchenko's result for Jordan algebras was proved by A. P. Semenov [21] in 1991. In particular, A. P. Semenov proved that if $J^{G}$ is a solvable algebra over a field of characteristic zero, then so is the Jordan algebra $J$. His proof uses a deep result by E.I. Zel'manov [22] which says that every Jordan nil-algebra of bounded index over a field of characteristic zero is solvable. If a Jordan algebra $J$ over a field of characteristic not equal to 2,3 admits an automorphism $\phi$ of the second order with solvable $J^{\phi}$, then $J$ is solvable [23].

In the case of alternative algebras one cannot expect that nilpotency of the invariant subalgebra implies the nilpotency of the whole algebra. There is an example (see [26, 27]) of a solvable non-nilpotent alternative algebra with an automorphism of order two such that its subalgebra of invariants is nilpotent. A combination of Semenov's result [21] and Zhevlakov's theorem [25] gives for an alternative algebra $A$ over a field of characteristic zero that the solvability of the algebra of invariants $A^{G}$ for a finite group $G$ implies the solvability of $A$. It is also known [24] that if $A$ is an alternative algebra over a field of characteristic not equal to 2 with an automorphism $\phi$ of order two, then the solvability of the algebra of invariants $A^{\phi}$ implies the solvability of $A$. In [28] M. Goncharov proved 
that an alternative $\mathbb{Z}_{3^{-}}$graded algebra $A=A_{0} \oplus A_{1} \oplus A_{3}$ over a field of characteristic not equal to $2,3,5$ is solvable if $A_{0}$ is solvable.

Notice that an algebra $A$ over a field containing all $n$th roots of unity admits an automorphism of order $n$ if and only if $A$ admits a $\mathbb{Z}_{n}$-grading.

In this paper we study the conditions of solvability of graded Novikov algebras from the point of view of the Bergman-Isaacs Theorem. We prove that a $\mathbb{Z}_{3}$-graded Novikov algebra $N=N_{0} \oplus N_{1} \oplus N_{2}$ over a field of characteristic not equal to 3 is solvable if $N_{0}$ is solvable. We also show that a $\mathbb{Z}_{2}$-graded Novikov algebra $N=N_{0} \oplus N_{1}$ over a field of characteristic not equal to 2 is solvable if $N_{0}$ is solvable. This implies that for every $n$ of the form $n=2^{k} 3^{l}$ that every $\mathbb{Z}_{n}$-graded Novikov algebra $N$ over a field of characteristic not equal to 2,3 is solvable if $N_{0}$ is solvable.

The paper is organized as follows. In Section 2 we give some preliminary facts. Section 3 is devoted to the study of $\mathbb{Z}_{2}$-graded Novikov algebras with solvable even part. In Section 4 we construct some ideals of $\mathbb{Z}_{3}$-graded Novikov algebras. The solvability of $\mathbb{Z}_{3}$-graded Novikov algebras with solvable 0-component is proven in Section 5.

\section{Preliminary calculations}

An algebra $N$ over a field $F$ is called a Novikov algebra if it satisfies the following identities:

$$
\begin{gathered}
(x, y, z)=(y, x, z) \text { (left symmetry), } \\
(x y) z=(x z) y \text { (right commutativity), }
\end{gathered}
$$

where $(x, y, z)=(x y) z-x(y z)$ is the associator of the elements $x, y, z$.

It follows from (2) that every Novikov algebra satisfies the identity

$$
(x y, z, t)=(x, z, t) y \text {. }
$$

Let $A$ be an arbitrary algebra and $X, Y, Z$ be subsets of $A$. Denote by $X Y$ the linear span of all products $x y$ and by $[X, Y]$ the linear span of all commutators $[x, y]=x y-y x$ where $x \in X, y \in Y$. Also denote by $(X, Y, Z)$ the linear span of all associators $(x, y, z)$ where $x \in X, y \in Y, z \in Z$.

Lemma 1. Let $N$ be a Novikov algebra, $B$ be its subalgebra, and $M, L$ be $B$-subbimodules of $N$. Then $B(M L),(M L) B \subseteq M L$, i.e., $M L$ is a B-bimodule. In particular, if $I, J$ are ideals of $N$, then $I J$ is an ideal of $N$ (see also [13]).

Proof. We have $(M L) B \subseteq(M B) L \subseteq M L$ by (2). By (1) we get

$$
B(M L) \subseteq(B M) L+(B, M, L) \subseteq M L+(M, B, L) \subseteq M L+(M B) L+M(B L) \subseteq M L
$$

since $M$ and $L$ are $B$-subbimodules of $N$.

Let $\mathbb{Z}_{n}=\mathbb{Z} / n \mathbb{Z}$ be the additive cyclic group of order $n$. Let

$$
N=N_{0} \oplus N_{1} \oplus N_{2} \oplus \ldots \oplus N_{n-1}, N_{i} N_{j} \subseteq N_{i+j}, i, j \in \mathbb{Z}_{n}
$$

be a $\mathbb{Z}_{n}$-graded Novikov algebra. Then the 0-component $N_{0}$ of $N$ is a subalgebra on $N$ and for convenience of notation we often denote this subalgebra by $A=N_{0}$. 
Lemma 2. Let $N$ be a $\mathbb{Z}_{n}$-graded Novikov algebra and $I$ be an ideal of $A$. Assume that

$$
\left(I \cdot N_{i}\right) N_{n-i},\left(N_{i} \cdot I\right) N_{n-i}, N_{n-i}\left(I \cdot N_{i}\right), N_{n-i}\left(N_{i} \cdot I\right) \subseteq I
$$

for some $i \in \mathbb{Z}_{n}$. Then

$$
\left(I^{2} \cdot N_{i}\right) N_{n-i},\left(N_{i} \cdot I^{2}\right) N_{n-i}, N_{n-i}\left(I^{2} \cdot N_{i}\right), N_{n-i}\left(N_{i} \cdot I^{2}\right) \subseteq I^{2} .
$$

Proof. Let $a, b \in I$ and $x \in N_{i}, y \in N_{n-i}$. We write $f \equiv g$ if $f-g \in I^{2}$. By Lemma 1 , $I^{2}$ is an ideal of $A$. Then

$$
\begin{aligned}
& ((a b) x) y \stackrel{\text { by }(2)}{=}((a x) y) b \equiv 0, \quad \text { since }(a x) y \in I \text {, i.e. }\left(I^{2} \cdot N_{i}\right) N_{n-i} \subseteq I^{2}, \\
& (x(a b)) y \stackrel{\text { by }(2)}{=}(x y)(a b) \equiv 0, \text { since } x y \in A \text {, i.e. }\left(N_{i} \cdot I^{2}\right) N_{n-i} \subseteq I^{2}, \\
& y((a b) x)=-(y, a b, x)+(y(a b)) x \stackrel{\text { by }(1) \text { and }(2)}{=}-(a b, y, x)+(y x)(a b) \equiv-(a b, y, x)=
\end{aligned}
$$

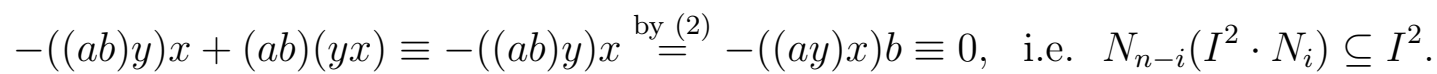

It remains to consider the product $y(x(a b))$. We have

$$
\begin{array}{r}
y(x(a b))=y\{-(x, a, b)+(x a) b\} \stackrel{\text { by }=(1)}{=}-y(a, x, b)+y((x a) b) \\
=-y((a x) b)+y(a(x b))-(y, x a, b)+(y(x a)) b \\
\stackrel{\text { by }(2)}{\equiv}-y((a b) x)-(y, a, x b)+(y a)(x b)-(x a, y, b) \\
\text { by }(1) \stackrel{\text { and }(2)}{\equiv}-(a, y, x b)+(y(x b)) a-((x a) y) b+(x a)(y b) \\
\stackrel{\text { by }(2)}{\equiv}-(a y)(x b)+a(y(x b))+(x(y b)) a \equiv-(a y)(x b) \\
=(a y, x, b)-((a y) x) b \equiv(a y, x, b) \stackrel{\text { by } \stackrel{(1)}{=}(x, a y, b)=(x(a y)) b-x((a y) b)}{=} \stackrel{(2)}{\equiv}-x((a b) y) \equiv 0 .
\end{array}
$$

Notice that in the last calculation we used twice that $y((a b) x) \equiv 0$, proven above.

The derived powers $A^{(s)}$ of an arbitrary algebra $A$ are defined by induction on $s$ as follows: $A^{(0)}=A$ and $A^{(s)}=A^{(s-1)} A^{(s-1)}$ for any positive integer $s \geq 1$. If $A$ is a Novikov algebra, then $A^{(s)}$ is an ideal of $A$ for all $s \geq 0$ by Lemma 1 . An algebra $A$ is called solvable if $A^{(s)}=0$ for some $s$. If $s$ is the minimal number such that $A^{(s)}=0$, then $s$ is called the length of solvability of $A$.

Corollary 1. Let $N$ be a $\mathbb{Z}_{n}$-graded Novikov algebra. Then

$$
\left(A^{(s)} \cdot N_{i}\right) N_{n-i},\left(N_{i} \cdot A^{(s)}\right) N_{n-i}, N_{n-i}\left(A^{(s)} \cdot N_{i}\right), N_{n-i}\left(N_{i} \cdot A^{(s)}\right) \subseteq A^{(s)}
$$

for any nonnegative integer $s$ and for any $i \in \mathbb{Z}_{n}$.

Proof. The statement of the corollary is obviously true for $s=0$. Assume that $s \geq 1$ and

$$
\left(A^{(s-1)} \cdot N_{i}\right) N_{n-i},\left(N_{i} \cdot A^{(s-1)}\right) N_{n-i}, N_{n-i}\left(A^{(s-1)} \cdot N_{i}\right), N_{n-i}\left(N_{i} \cdot A^{(s-1)}\right) \subseteq A^{(s-1)} .
$$

Since $A^{(s)}=A^{(s-1)} A^{(s-1)}$ and $A^{(s-1)}$ is an ideal of $A$, it follows from Lemma 2 that

$$
\left(A^{(s)} \cdot N_{i}\right) N_{n-i},\left(N_{i} \cdot A^{(s)}\right) N_{n-i}, N_{n-i}\left(A^{(s)} \cdot N_{i}\right), N_{n-i}\left(N_{i} \cdot A^{(s)}\right) \subseteq A^{(s)}
$$




\section{3. $\mathbb{Z}_{2}$-GRADED Novikov ALGEBRA With SOlvable EVEN PART}

If $N$ is a $\mathbb{Z}_{2}$-graded Novikov algebra, then we write $N=A \oplus M$ by setting $A=N_{0}$ and $M=N_{1}$. Notice that the study of $\mathbb{Z}_{2}$-graded algebras is more popular since it is related to the study of superalgebras. In this case $A$ is called the even part and $M$ is called the odd part of $N$.

In this section we prove that every Novikov algebra over a field of characteristic $\neq 2$ with solvable even part is solvable. First formulate one important corollary of Lemma 2.

Corollary 2. Let $N=A \oplus M$ be a $\mathbb{Z}_{2}$-graded Novikov algebra. Then

$$
J=A^{2} \oplus\left(A^{2} M+M A^{2}\right)
$$

is a $\mathbb{Z}_{2}$-graded ideal of $N$.

Proof. We write $f \equiv g$ if $f-g \in J$. Let $a, b, c \in A$ and $m, n \in M$. First we prove that $J$ is a right ideal of $N$. We have

$$
\begin{aligned}
(a b) c \equiv 0,(a b) n & \equiv 0, \\
((a b) m) c \stackrel{\text { by }(2)}{=}((a b) c) m & \equiv 0, \\
(m(a b)) c \stackrel{\text { by }(2)}{=}(m c)(a b) & \equiv 0,
\end{aligned}
$$

since $m c \in M$. By Lemma 2, $((a b) m) n,(m(a b)) n \in A^{2}$.

Now we prove that $J$ is a left ideal. We have

$$
\begin{aligned}
c(a b) \equiv 0, n(a b) & \equiv 0, \\
c((a b) m)=-(c, a b, m)+(c(a b)) m \stackrel{\text { by }(1)}{\equiv}-(a b, c, m)=-((a b) c) m+(a b)(c m) & \equiv 0, \\
c(m(a b))=-(c, m, a b)+(c m)(a b) \stackrel{\text { by }(1)}{\equiv}-(m, c, a b)=-(m c)(a b)+m(c(a b)) & \equiv 0 .
\end{aligned}
$$

By Lemma $2 n((a b) m), n(m(a b)) \in A^{2}$.

Thus $J$ is a $\mathbb{Z}_{2}$-graded ideal of $N$.

Proposition 1. Let $F$ be a field of characteristic $\neq 2$ and let $N=A \oplus M$ be a $\mathbb{Z}_{2}$-graded Novikov algebra. Suppose that $A^{2}=0$. Then $N^{(n)}=0$ for some positive integer $n$.

Several lemmas precede the proof of this proposition. These lemmas are formulated under the conditions of Proposition 1.

Lemma 3. Let $I=M M+A M$. Then $I$ is a $\mathbb{Z}_{2}$-graded ideal of algebra $N^{2}$.

Proof. It is clear that $N^{2}=M M+A M+M A$. Then $M^{2} N^{2} \subseteq A M \subseteq I$. Since $A^{2}=0$, using (2) we get

Hence

$$
(A M) M^{2} \subseteq\left(A M^{2}\right) M=0 .
$$

$$
I N^{2} \subseteq M^{2} N^{2}+(A M) M^{2}+M^{2} \subseteq I .
$$

Therefore, $I$ is a right ideal of $N^{2}$.

Now we prove that $I$ is a left ideal of $N^{2}$. By (1) we have

$$
(M A) M^{2} \subseteq\left(M, A, M^{2}\right)+M\left(A M^{2}\right) \subseteq \underset{5}{\left(A, M, M^{2}\right) \subseteq(A M) M^{2}+A\left(M M^{2}\right) \subseteq A M .}
$$


Hence $N^{2} M^{2} \subseteq I$. From here we obtain that

$$
(A M+M A) I \subseteq(A M+M A) M^{2}+(A M+M A)(A M) \subseteq N^{2} M^{2}+M^{2} \subseteq I .
$$

Therefore,

$$
N^{2} I \subseteq M^{2} I+(A M+M A) I \subseteq I .
$$

Thus $I$ is a $\mathbb{Z}_{2}$-graded ideal of the algebra $N^{2}$.

Lemma 4. We can assume that $M A=0$.

Proof. Let $I=M^{2}+A M$. Since $N^{2}=I+M A$, applying Lemma 3 we get

$$
\left(N^{2}\right)^{2}=(I+M A)^{2} \subseteq I^{2}+I(M A)+(M A) I+(M A)^{2} \subseteq I .
$$

Hence, the algebra $N$ is solvable if the algebra $I=M M+A M$ is solvable. By (2), we have

$$
(A M) M^{2} \subseteq\left(A M^{2}\right) M=0 .
$$

Considering $I$ instead of $N$, we can assume that $M A=0$.

Lemma 5. We can assume that $x y=y x$ for all $x, y \in M$.

Proof. Let $x, y \in M$. First we show that $[x, y] M=0$. Let $z \in M$. By lemma 4, we obtain

$$
(x y) z=(x, y, z)+x(y z)=(x, y, z) \stackrel{\text { by }(1)}{=}(y, x, z)=(y x) z
$$

since $y z, x z \in A$. Therefore, $[x, y] M=0$ for all $x, y \in M$. By Lemma 4, we have $M[x, y]=0$, since $[x, y] \in A$.

Hence, the vector space $[M, M]$ spanned by all commutators $[x, y]$, where $x, y \in M$, lies in the annihilator of the algebra $N$. Therefore, the algebra $N$ is solvable, if the quotient algebra $N /[M, M]$ of $N$ is a solvable algebra. Changing $N$ to $N /[M, M]$, we can assume that $x y=y x$ for all $x, y \in M$.

The proof of Proposition 1. By Lemma 4, we can assume that $M A=0$. Let $I=$ $M M+A M$. Then $N^{2}=I$. Therefore, it is sufficient to prove that the algebra $I$ is solvable.

Let $a, b \in A, x, y \in M$. By (2), we have $(a x)(b y)=(a(b y)) x$. Using (1) and $M A=0$ we obtain that

$$
(a(b y)) x=(a, b y, x)+a((b y) x)=(b y, a, x)=((b y) a) x-(b y)(a x)=-(b y)(a x) .
$$

Therefore, $(a x)(b y)=-(b y)(a x)$. By Lemma $5, x y=y x$ for all $x, y \in M$. Using this we get $(a x)(b y)=0$ over a field of characteristic $\neq 2$.

Consequently,

$$
I^{2} \subseteq(M M)^{2}+(A M)^{2}+(M M)(A M) \subseteq A M
$$

Hence $I^{(2)}=0$.

Thus the algebra $N$ is solvable.

Theorem 1. Every $\mathbb{Z}_{2}$-graded Novikov algebra with solvable even part over a field of characteristic $\neq 2$ is solvable. 
Proof. Let $N=A \oplus M$ be a $\mathbb{Z}_{2}$-graded Novikov algebra with solvable even part $A$. Let $n$ be the length of solvability of $A$. We prove the statement of the theorem by induction on $n$. If $n=1$, then $N$ is solvable by Proposition 1 .

By Corollary $2, J=A^{2} \oplus\left(A^{2} M+M A^{2}\right)$ is a $\mathbb{Z}_{2}$-graded ideal of $N$. Notice that the even part $A^{2}$ of $J$ is solvable with solvability length $n-1$. By the induction proposition, $J$ is solvable, that is, $J^{(s)}=0$ for some positive integer $s$. Moreover, the even part of the quotient algebra $\bar{N}=N / J=A / A^{2} \oplus M /\left(A^{2} M+M A^{2}\right)$ has trivial multiplication. Consequently, $\bar{N}$ is solvable by Proposition 1 , that is, $N^{(t)} \subseteq J$ for some positive integer $t$. Then, $N^{(s+t)}=0$.

Recall that the powers of an arbitrary algebra $A$ are defined inductively by $A^{1}=A$ and $A^{n}=\sum_{i=1}^{n} A^{i} A^{n-i}$ for all integers $n \geq 2$. An algebra $A$ is called nilpotent if $A^{n}=0$ for some positive integer $n$. Obviously, every nilpotent algebra is solvable. The converse is not true in the case of Novikov algebras.

Example 1. [13] Let $N=F a+F b$ be a vector space of dimension 2. The product on $N$ is defined as

$$
a b=b, a^{2}=b^{2}=b a=0 .
$$

It is easy to check that $N$ is a solvable Novikov algebra but not nilpotent since $a(a \ldots(a b) \ldots)=$ $b \neq 0$.

Moreover, $N$ is a $\mathbb{Z}_{2}$-graded Novikov algebra with $N_{0}=F a$ and $N_{1}=F b$. The even part $N_{0}$ of $N$ is nilpotent. This means that in the formulation of Theorem 1, solvability cannot be replaced by nilpotency.

\section{Some ideals of $\mathbb{Z}_{3}$-GRAded Novikov AlgeBras}

Let

$$
N=N_{0} \oplus N_{1} \oplus N_{2}
$$

be a $\mathbb{Z}_{3}$-graded Novikov algebra. We fix a a $\mathbb{Z}_{3}$-graded subspace

$$
I=I_{0} \oplus I_{1} \oplus I_{2}
$$

where

$$
I_{0}=A^{2}+N_{1} N_{2}+N_{2} N_{1}, I_{1}=A^{2} N_{1}+N_{1} A^{2}+N_{2}^{2}, I_{2}=A^{2} N_{2}+N_{2} A^{2}+N_{1}^{2} .
$$

Lemma 6. Let $N$ be a $\mathbb{Z}_{3}$-graded Novikov algebra. Then the subspace I from (4) is a $\mathbb{Z}_{3}$-graded ideal of $N^{2}$. Moreover, $N^{(2)} \subseteq I$.

Proof. Since $A, N_{0}$, and $N_{2}$ are $A$-bimodules, then $I$ is also an $A$-bimodule by Lemma 1 , that is $I A \subseteq I$ and $A I \subseteq I$. Moreover, since $N_{2}^{2}+A^{2} N_{1}+N_{1} A^{2} \subseteq N_{1}$ and $N_{1}^{2}+A^{2} N_{2}+$ $N_{2} A^{2} \subseteq N_{2}$, it follows that $I$ is a subalgebra of $N$.

We first prove the inclusions

$$
I\left(A N_{i}\right), I\left(N_{i} A\right),\left(A N_{i}\right) I,\left(N_{i} A\right) I \subseteq I, \quad i=1,2 .
$$

We will check only the inclusion $I\left(A N_{1}\right) \subseteq I$ since the other inclusions can be checked similarly. We have $\left(N_{1} N_{2}\right) N_{1} \subseteq\left(N_{1} N_{1}\right) N_{2} \subseteq N_{2}^{2}$ by (2). Therefore, $\left(N_{1} N_{2}\right)\left(A N_{1}\right) \subseteq$ 
$N_{2}^{2} \subseteq I$. By (2) and (1) we have

$$
\begin{aligned}
& \left(N_{2} N_{1}\right)\left(A N_{1}\right) \subseteq\left(N_{2} \cdot A N_{1}\right) N_{1} \subseteq\left(N_{2}, A N_{1}, N_{1}\right)+N_{2} N_{1}^{2} \\
\subseteq & \left(A N_{1}, N_{2}, N_{1}\right)+N_{2}^{2} \subseteq\left(A N_{1} \cdot N_{2}\right) N_{1}+\left(A N_{1}\right)\left(N_{2} N_{1}\right)+N_{2}^{2} \\
\subseteq & \left(A N_{1} \cdot N_{1}\right) N_{2}+\left(A \cdot N_{2} N_{1}\right) N_{1}+N_{2}^{2} \subseteq N_{2}^{2}+A^{2} N_{2} \subseteq I .
\end{aligned}
$$

These inclusions imply that

$$
I_{0}\left(A N_{1}\right)=\left(A^{2}+N_{1} N_{2}+N_{2} N_{1}\right)\left(A N_{1}\right) \subseteq I
$$

since $\left(A^{2}\right)\left(A N_{1}\right) \subseteq\left(A^{2}\right) N_{1} \subseteq I$. Notice that $I_{1}\left(A N_{1}\right) \subseteq N_{1}\left(A N_{1}\right) \subseteq N_{1}^{2} \subseteq I$. Moreover, $\left(A^{2} N_{2}+N_{2} A^{2}\right)\left(A N_{1}\right) \subseteq A^{2} \subseteq I$ by Corollary 1. Since $N_{1}^{2}\left(A N_{1}\right) \subseteq N_{2} N_{1} \subseteq I$ it follows that $I_{2}\left(A N_{1}\right) \subseteq I$. Consequently, $I\left(A N_{1}\right) \subseteq I$.

Notice that

$$
N^{2}=\left(A^{2}+N_{1} N_{2}+N_{2} N_{1}\right) \oplus\left(A N_{1}+N_{1} A+N_{2}^{2}\right) \oplus\left(A N_{2}+N_{2} A+N_{1}^{2}\right)
$$

and

$$
N^{2}=I+A N_{1}+N_{1} A+A N_{2}+N_{2} A .
$$

Since $I$ is a subalgebra of $N$, the inclusions (5) imply that $I$ is an ideal of $N^{2}$. It is clear that $N^{(2)} \subseteq I$.

Lemma 7. Let $N$ be a $\mathbb{Z}_{3}$-graded Novikov algebra. Let

$$
K=A^{2} \oplus\left(A^{2} N_{1}+N_{1} A^{2}+\left(A^{2} N_{2}\right)^{2}\right) \oplus\left(A^{2} N_{2}+N_{2} A^{2}+\left(A^{2} N_{1}\right)^{2}\right)
$$

be a $\mathbb{Z}_{3}$-graded subspace of $I$. Then $K$ is a $\mathbb{Z}_{3}$-graded ideal of $I$.

Proof. We have $K A, A K \subseteq K$ by Lemma 1 . First we show that

$$
N\left(A^{2} N_{i}\right)^{2},\left(A^{2} N_{i}\right)^{2} N \subseteq K, \quad i=1,2 .
$$

We check these inclusions for $i=1$ since the case $i=2$ can be treated similarly. An obvious inclusion $A^{2} N_{1} \cdot N_{1} \subseteq N_{2},(2)$, and Corollary 1 imply that

$$
\left(A^{2} N_{1}\right)^{2} N_{1} \subseteq\left(A^{2} N_{1} \cdot A^{2} N_{1}\right) N_{1} \subseteq\left(A^{2} N_{1} \cdot N_{1}\right) \cdot A^{2} N_{1} \subseteq N_{2} \cdot A^{2} N_{1} \subseteq A^{2} \subseteq K .
$$

By (1), we also get

$$
\begin{array}{r}
N_{1}\left(A^{2} N_{1}\right)^{2} \subseteq\left(N_{1}, A^{2} N_{1}, A^{2} N_{1}\right)+\left(N_{1} \cdot A^{2} N_{1}\right) \cdot A^{2} N_{1} \\
\subseteq\left(A^{2} N_{1}, N_{1}, A^{2} N_{1}\right)+N_{2} \cdot A^{2} N_{1} \\
\subseteq\left(A^{2} N_{1} \cdot N_{1}\right) \cdot A^{2} N_{1}+A^{2} N_{1} \cdot\left(N_{1} \cdot A^{2} N_{1}\right)+N_{2} \cdot A^{2} N_{1} \subseteq N_{2}\left(A^{2} N_{1}\right)+\left(A^{2} N_{1}\right) N_{2}
\end{array}
$$

since $A^{2} N_{1} \cdot N_{1}, N_{1} \cdot A^{2} N_{1} \subseteq N_{2}$. Applying Corollary 1 we obtain $N_{1}\left(A^{2} N_{1}\right)^{2} \subseteq A^{2} \subseteq K$. Notice that $A^{2} N_{1} \cdot N_{2} \subseteq A^{2}$ by Corollary 1 . Using (2) we get

$$
\left(A^{2} N_{1}\right)^{2} N_{2} \subseteq\left(A^{2} N_{1} \cdot N_{2}\right) \cdot A^{2} N_{1} \subseteq A^{2} \cdot A^{2} N_{1} \subseteq A^{2} N_{1} \subseteq K .
$$

Similarly,

$$
\begin{array}{r}
N_{2}\left(A^{2} N_{1}\right)^{2} \subseteq\left(N_{2}, A^{2} N_{1}, A^{2} N_{1}\right)+\left(N_{2} \cdot A^{2} N_{1}\right) \cdot A^{2} N_{1} \\
\subseteq\left(A^{2} N_{1}, N_{2}, A^{2} N_{1}\right)+A^{2} \cdot A^{2} N_{1} \\
\subseteq\left(A^{2} N_{1} \cdot N_{2}\right) \cdot A^{2} N_{1}+A^{2} N_{1} \cdot\left(N_{2} \cdot A^{2} N_{1}\right)+A^{2} \cdot A^{2} N_{1} \\
\subseteq A^{2} \cdot A^{2} N_{1}+A^{2} N_{1} \cdot A^{2} \subseteq K . \\
8
\end{array}
$$


We now prove that $K$ is a subalgebra of $N$. By Corollary 1,

$$
\left(A^{2} N_{1}+N_{1} A^{2}\right) N_{2}, N_{2}\left(A^{2} N_{1}+N_{1} A^{2}\right) \subseteq A^{2} .
$$

Consequently,

$$
\left(A^{2} N_{1}+N_{1} A^{2}\right)\left(A^{2} N_{2}+N_{2} A^{2}\right),\left(A^{2} N_{2}+N_{2} A^{2}\right)\left(A^{2} N_{1}+N_{1} A^{2}\right) \subseteq A^{2} .
$$

Using (2) we get

$$
\left(N_{1} A^{2}\right)\left(A^{2} N_{1}\right) \subseteq\left(N_{1} \cdot A^{2} N_{1}\right) A^{2} \subseteq N_{2} A^{2}, \quad\left(N_{1} A^{2}\right)\left(N_{1} A^{2}\right) \subseteq N_{2} A^{2} .
$$

Similarly,

$$
\left(A^{2} N_{1}\right)\left(N_{1} A^{2}\right) \subseteq\left(A^{2} \cdot N_{1} A^{2}\right) N_{1} \subseteq\left(A^{2} N_{1} \cdot A^{2}+\left(A^{2}, N_{1}, A^{2}\right)\right) N_{1} .
$$

Applying (2), (1), and (3), we obtain

$$
\begin{gathered}
\left(A^{2} N_{1}\right)\left(N_{1} A^{2}\right) \subseteq\left(A^{2} N_{1} \cdot A^{2}+\left(A^{2}, N_{1}, A^{2}\right)\right) N_{1} \subseteq\left(A^{2} N_{1} \cdot N_{1}\right) A^{2}+\left(N_{1}, A^{2}, A^{2}\right) N_{1} \subseteq \\
N_{2} A^{2}+\left(N_{1} N_{1}, A^{2}, A^{2}\right) \subseteq N_{2} A^{2} .
\end{gathered}
$$

Similarly, $\left(N_{2} A^{2}\right)\left(A^{2} N_{2}\right),\left(N_{2} A^{2}\right)\left(N_{2} A^{2}\right),\left(A^{2} N_{2}\right)\left(N_{2} A^{2}\right) \subseteq N_{1} A^{2}$.

These inclusions together with (6) give that $K$ is a subalgebra of $N$.

We now prove that $K$ is an ideal of $I$. It is clear that

$$
\left(N_{1}^{2}+N_{2}^{2}\right) A^{2}, A^{2}\left(N_{1}^{2}+N_{2}^{2}\right) \subset K .
$$

By Corollary 1, we get

$$
\left(A^{2} N_{1}+N_{1} A^{2}\right) N_{1}^{2}, N_{1}^{2}\left(A^{2} N_{1}+N_{1} A^{2}\right) \subseteq A^{2}
$$

since $N_{1}^{2} \subseteq N_{2}$. Using (2) and Corollary 1 we also get

$$
N_{2}^{2}\left(A^{2} N_{1}+N_{1} A^{2}\right) \subseteq\left(N_{2} \cdot\left(A^{2} N_{1}+N_{1} A^{2}\right) N_{2} \subseteq A^{2} N_{2} .\right.
$$

Similar calculations with (1) give that

$$
\begin{gathered}
\left(A^{2} N_{1}+N_{1} A^{2}\right) N_{2}^{2} \subseteq\left(A^{2} N_{1}+N_{1} A^{2}, N_{2}, N_{2}\right)+\left(\left(A^{2} N_{1}+N_{1} A^{2}\right) N_{2}\right) N_{2} \subseteq \\
\left(N_{2}, A^{2} N_{1}+N_{1} A^{2}, N_{2}\right)+A^{2} N_{2} \subseteq A^{2} N_{2}+N_{2} A^{2} \subseteq K .
\end{gathered}
$$

Therefore,

$$
I\left(A^{2} N_{1}+N_{1} A^{2}\right),\left(A^{2} N_{1}+N_{1} A^{2}\right) I \subseteq K
$$

Similarly,

$$
I\left(A^{2} N_{2}+N_{2} A^{2}\right),\left(A^{2} N_{2}+N_{2} A^{2}\right) I \subseteq K .
$$

These inclusions together with (6) give that $K$ is an ideal of $I$. 


\section{5. $\mathbb{Z}_{3}$-GRADED Novikov Algebras With SOlvable 0-COMPONENT}

In this section we show that $\mathbb{Z}_{3}$-graded Novikov algebras over a field of characteristic $\neq 3$ with solvable 0 -component are solvable. We start with the case when the length of solvability of the 0-component is 1 .

Proposition 2. Let $F$ be a field of characteristic $\neq 3$ and let $N=N_{0} \oplus N_{1} \oplus N_{2}$ be a $\mathbb{Z}_{3}$-graded Novikov algebra. Suppose that $N_{0}^{2}=0$. Then $N$ is a solvable algebra.

We give several lemmas prior to the proof of this proposition. These lemmas are formulated under the conditions of Proposition 2. The 0-component of $N$ is usually denoted by $A=N_{0}$.

First formulate a direct corollary of Lemma 6 .

Corollary 3. The vector space $I=N_{1} N_{2}+N_{2} N_{1}+N_{1}^{2}+N_{2}^{2}$ is a $\mathbb{Z}_{3}$-graded ideal of $N^{2}$. Moreover, $N^{(2)} \subseteq I$.

For any elements $a, b \in N$ define $a \circ b=a b+b a$.

Lemma 8. Let $a \in N_{1}^{2}$ and $b \in N_{2}^{2}$. Then $a \circ b=0$. Moreover, we can assume that $x \circ y=0$ for any $x \in N_{1}, y \in N_{2}$ and $A=N_{1} N_{2}$.

Proof. Let $x_{1}, y_{1} \in N_{1}$ and $x_{2}, y_{2} \in N_{2}$. Then

$$
\begin{array}{r}
\left(x_{1} y_{1}\right)\left(x_{2} y_{2}\right) \stackrel{\text { by }}{=}\left(x_{1} \cdot x_{2} y_{2}\right) y_{1}=\left(x_{1} x_{2} \cdot y_{2}\right) y_{1}-\left(x_{1}, x_{2}, y_{2}\right) y_{1} \\
\stackrel{\text { by }(1)}{=}\left(x_{1} x_{2} \cdot y_{2}\right) y_{1}-\left(x_{2}, x_{1}, y_{2}\right) y_{1}=\left(x_{1} x_{2} \cdot y_{2}\right) y_{1}-\left(x_{2} x_{1} \cdot y_{2}\right) y_{1}+\left(x_{2} \cdot x_{1} y_{2}\right) y_{1} \\
\stackrel{\text { by }}{=}\left(\left[x_{1}, x_{2}\right] y_{2}\right) y_{1}+\left(x_{2} y_{1}\right)\left(x_{1} y_{2}\right)=\left(\left[x_{1}, x_{2}\right] y_{2}\right) y_{1}
\end{array}
$$

since $\left(x_{2} y_{1}\right)\left(x_{1} y_{2}\right)=0$. Similarly, $\left(x_{2} y_{2}\right)\left(x_{1} y_{1}\right)=\left(\left[x_{2}, x_{1}\right] y_{1}\right) y_{2}$.

Therefore, we have

$$
\left(x_{1} y_{1}\right) \circ\left(x_{2} y_{2}\right)=\left(\left[x_{1}, x_{2}\right] y_{2}\right) y_{1}+\left(\left[x_{2}, x_{1}\right] y_{1}\right) y_{2} \stackrel{\text { by }}{=}(2)\left(\left(\left[x_{1}, x_{2}\right]+\left[x_{2}, x_{1}\right]\right) y_{2}\right) y_{1}=0 .
$$

Consequently, $a \circ b=0$ for all $a \in N_{1}^{2}, b \in N_{2}^{2}$.

By Corollary 3, $I$ is an ideal of $N^{2}$ and $N^{(2)} \subseteq I$. Consequently, the algebra $N$ is solvable if and only if $I$ is solvable. Replacing $N$ by $I$, we may assume that $x \circ y=0$ for all $x \in N_{1}, y \in N_{2}$ and $A=N_{1} N_{2}$.

Lemma 9. The following equalities hold in $N$ :

$$
\left[N_{1}, N_{1}\right] N_{2}=0,\left[N_{2}, N_{2}\right] N_{1}=0,\left(N_{1} N_{2}\right)\left[N_{i}, N_{i}\right]=0,\left[N_{i}, N_{i}\right]\left(N_{1} N_{2}\right)=0,
$$

where $i=1,2$.

Proof. Let $x_{1}, y_{1} \in N_{1}$ and $x_{2} \in N_{2}$. By Lemma 8, $x_{1} x_{2}=-x_{2} x_{1}$ and $y_{1} x_{2}=-x_{2} y_{1}$. Then we obtain

$$
\left(x_{1} y_{1}\right) x_{2} \stackrel{\text { by }}{=}\left(x_{1} x_{2}\right) y_{1}=-\left(x_{2} x_{1}\right) y_{1} \stackrel{\text { by }}{=}-\left(x_{2} y_{1}\right) x_{1}=\left(y_{1} x_{2}\right) x_{1} \stackrel{\text { by }}{=}(2)\left(y_{1} x_{1}\right) x_{2} .
$$

Therefore, $\left[x_{1}, y_{1}\right] x_{2}=0$. Hence, $\left[N_{1}, N_{1}\right] N_{2}=0$. Similarly, $\left[N_{2}, N_{2}\right] N_{1}=0$.

Now we will show that $\left(N_{1} N_{2}\right)\left[N_{1}, N_{1}\right]=0$. By $(2)$,

$$
\left(N_{1} N_{2}\right)\left[N_{1}, N_{1}\right] \subseteq\left(N_{10}\left[N_{1}, N_{1}\right]\right) N_{2}
$$


Since $\left[N_{1}, N_{1}\right] \subseteq N_{2}$ and $N_{1} N_{2} \subseteq N_{2} N_{1}$ by Lemma 8, it follows that

$$
\left(N_{1} N_{2}\right)\left[N_{1}, N_{1}\right] \subseteq\left(N_{1}\left[N_{1}, N_{1}\right]\right) N_{2} \subseteq\left(\left[N_{1}, N_{1}\right] N_{1}\right) N_{2} \subseteq\left(\left[N_{1}, N_{1}\right] N_{2}\right) N_{1}=0 .
$$

Notice that

$$
\left[N_{1}, N_{1}\right]\left(N_{1} N_{2}\right) \subseteq\left(\left[N_{1}, N_{1}\right], N_{1}, N_{2}\right)+\left(\left[N_{1}, N_{1}\right] N_{1}\right) N_{2} .
$$

From this, using (1) and (2), we get

$$
\begin{aligned}
{\left[N_{1}, N_{1}\right]\left(N_{1} N_{2}\right) } & \subseteq\left(N_{1},\left[N_{1}, N_{1}\right], N_{2}\right)+\left(\left[N_{1}, N_{1}\right] N_{2}\right) N_{1} \\
& \subseteq\left(N_{1},\left[N_{1}, N_{1}\right], N_{2}\right) \subseteq\left(N_{1}\left[N_{1}, N_{1}\right]\right) N_{2},
\end{aligned}
$$

since $\left[N_{1}, N_{1}\right] N_{2}=0$. Since $\left(N_{1} N_{2}\right)\left[N_{1}, N_{1}\right]=0$, applying (2) we obtain

$$
\left[N_{1}, N_{1}\right]\left(N_{1} N_{2}\right) \subseteq\left(N_{1}\left[N_{1}, N_{1}\right]\right) N_{2} \subseteq\left(N_{1} N_{2}\right)\left[N_{1}, N_{1}\right]=0 .
$$

Similarly, $\left(N_{1} N_{2}\right)\left[N_{2}, N_{2}\right]=0$ and $\left[N_{2}, N_{2}\right]\left(N_{1} N_{2}\right)=0$.

Lemma 10. We can assume that $N_{1} A=0, N_{2} A=0$.

Proof. First we prove that $K=N_{1} N_{2}+A N_{1}+N_{1} A+N_{1}^{2}$ is a $\mathbb{Z}_{3}$-graded ideal of the algebra $N$ and $N^{(3)} \subseteq K$.

We have $A N_{1}^{2}, N_{1}^{2} \bar{A} \subseteq N_{1}^{2}$ by Lemma 1. Hence $A K, K A \subseteq K$. Since $N_{1}^{2} \subseteq N_{2}$ it follows that $N_{1} K, K N_{1} \subseteq K$. By Lemma 8 and (2), we get

$$
\left(N_{1} N_{2}\right) N_{2} \subseteq\left(N_{2} N_{1}\right) N_{2} \subseteq N_{2}^{2} N_{1} \subseteq N_{1} N_{1}
$$

Therefore, $K N_{2} \subseteq K$, since $N_{1}^{2} N_{2} \subseteq\left(N_{1} N_{2}\right) N_{1} \subseteq A N_{1}$.

Applying (1) and (2), we see that

$$
\begin{gathered}
N_{2}\left(N_{1} N_{2}\right) \subseteq\left(N_{2} N_{1}\right) N_{2}+\left(N_{2}, N_{1}, N_{2}\right) \subseteq\left(N_{2} N_{2}\right) N_{1}+\left(N_{2}, N_{1}, N_{2}\right) \subseteq \\
N_{1}^{2}+\left(N_{1}, N_{2}, N_{2}\right) \subseteq N_{1}^{2}+\left(N_{1} N_{2}\right) N_{2}+N_{1} N_{2}^{2} \subseteq N_{1}^{2} .
\end{gathered}
$$

Similarly, one can prove that

$$
N_{2} N_{1}^{2} \subseteq\left(N_{2} N_{1}\right) N_{1}+\left(N_{2}, N_{1}, N_{1}\right) \subseteq A N_{1}+\left(N_{1}, N_{2}, N_{1}\right) \subset A N_{1}+N_{1} A .
$$

Therefore, $N_{2} K \subset K$.

Consequently, $K$ is a $\mathbb{Z}_{3}$-graded ideal of the algebra $N$.

Let $N / K=\bar{A}+\overline{N_{1}}+\overline{N_{2}}$, where $\bar{A}, \overline{N_{1}}, \overline{N_{2}}$ are the images of $A, N_{1}, N_{2}$ in the quotient algebra $N / K$, respectively. Then $(N / K)^{2} \subseteq{\overline{N_{2}}}^{2}+\overline{N_{2}} \subseteq \overline{N_{1}}+\overline{N_{2}}$. Therefore, $(N / K)^{(2)} \subseteq$ ${\overline{N_{2}}}^{2} \subseteq \overline{N_{1}}$. Hence $N^{(3)} \subseteq K$ and $N$ is a solvable if and only if $K$ is solvable.

Let $K_{1}=N_{1} N_{2}+A N_{1}+N_{1}^{2}$. We show that $K_{1}$ is an ideal of $K$.

We have $N_{1}^{2} N_{1}^{2} \subseteq\left(N_{1} N_{1}^{2}\right) N_{1} \subseteq A N_{1}$ by (2). By Lemma 1 , we get $A K_{1}, K_{1} A \subseteq K_{1}$. Therefore, $K_{1}^{2} \subseteq K_{1}$, i.e., $K_{1}$ is a subalgebra of $K$. Using (1) and (2), we also have

$$
\left(N_{1} A\right) A=\left(N_{1}, A, A\right) \subseteq\left(A, N_{1}, A\right) \subseteq\left(A N_{1}\right) A+A\left(N_{1} A\right) \subseteq A^{2} N_{1}+A N_{1} \subseteq A N_{1} .
$$

Therefore, $K_{1}$ is a ideal of $K$. Moreover, $K^{2} \subseteq K_{1}$. Hence the algebra $N$ is solvable if and only if $K_{1}$ is solvable. Therefore, replacing $N$ by $K_{1}$, we can assume that $N_{1} A=0$ since $\left(A N_{1}\right) A \subseteq A^{2} N_{1}=0$. In this case we have $N_{1}^{2} A \subseteq\left(N_{1} A\right) N_{1}=0$.

Thus, we can assume that $N_{1} A=0, N_{2} A=0$ in $N$.

Lemma 11. We can assume that $\left[N_{1}, N_{1}\right]=\left[N_{2}, N_{2}\right]=0$. 
Proof. First we prove that the vector space $K=N_{1}\left[N_{1}, N_{1}\right]+N_{2}\left[N_{1}, N_{1}\right]+\left[N_{1}, N_{1}\right]$ is a $\mathbb{Z}_{3}$-graded ideal of $N$ and $K^{(2)}=0$.

We have $A=N_{1} N_{2}$ by Lemma 8. Then Lemma 9 and Lemma 1 give that $A K, K A \subseteq K$.

We prove that $N_{1} K \subseteq K$. By Lemma 10, we have $N_{1}\left(N_{1}\left[N_{1}, N_{1}\right]\right) \subseteq N_{1} A=0$. Since $N_{2}\left[N_{1}, N_{1}\right] \subseteq N_{1}$, using (2) and Lemma 9 we get

$$
\begin{array}{r}
N_{1}\left(N_{2}\left[N_{1}, N_{1}\right]\right) \subseteq\left[N_{1}, N_{2}\left[N_{1}, N_{1}\right]\right]+\left(N_{2}\left[N_{1}, N_{1}\right]\right) N_{1} \\
\subseteq\left[N_{1}, N_{1}\right]+\left(N_{2} N_{1}\right)\left[N_{1}, N_{1}\right] \subseteq\left[N_{1}, N_{1}\right] .
\end{array}
$$

Therefore, $N_{1} K \subseteq K$.

Applying (2), Lemma 9, and Lemma 8, we obtain that

$$
\begin{array}{r}
K N_{1} \subseteq N_{1}^{2}\left[N_{1}, N_{1}\right]+\left(N_{2} N_{1}\right)\left[N_{1}, N_{1}\right]+\left[N_{1}, N_{1}\right] N_{1} \\
\subseteq N_{2}\left[N_{1}, N_{1}\right]+N_{1}\left[N_{1}, N_{1}\right] \subseteq K .
\end{array}
$$

Consequently, $N_{1} K \subseteq K$ and $K N_{1} \subseteq K$.

We prove that $N_{2} K, K N_{2} \subseteq K$. By Lemma 10, Lemma 8, and (2), we get

$$
\begin{array}{r}
N_{2} K \subseteq\left(N_{2}\left[N_{1}, N_{1}\right]\right) N_{2}+N_{2}\left[N_{1}, N_{1}\right] \\
\subseteq N_{2}^{2}\left[N_{1}, N_{1}\right]+N_{2}\left[N_{1}, N_{1}\right] \subseteq N_{1}\left[N_{1}, N_{1}\right]+N_{2}\left[N_{1}, N_{1}\right] \subseteq K .
\end{array}
$$

Since $\left[N_{1}, N_{1}\right] N_{2}=0$ and $\left(N_{1} N_{2}\right)\left[N_{1}, N_{1}\right]=0$ by Lemma 9 , using (2) we obtain

$$
\begin{gathered}
K N_{2} \subseteq\left(N_{1}\left[N_{1}, N_{1}\right]\right) N_{2}+\left(N_{2}\left[N_{1}, N_{1}\right]\right) N_{2}+\left[N_{1}, N_{1}\right] N_{2} \subseteq \\
\left(N_{1} N_{2}\right)\left[N_{1}, N_{1}\right]+N_{2}^{2}\left[N_{1}, N_{1}\right] \subseteq N_{1}\left[N_{1}, N_{1}\right] .
\end{gathered}
$$

Therefore, $K$ is an ideal of the algebra $N$.

Applying Lemma 9, (1), and Lemma 10, we also get

$$
\begin{gathered}
N_{1}\left(N_{2}\left[N_{1}, N_{1}\right]\right) \subseteq\left(N_{1}, N_{2},\left[N_{1}, N_{1}\right]\right) \subseteq\left(N_{2}, N_{1},\left[N_{1}, N_{1}\right]\right) \subseteq \\
\left(N_{2} N_{1}\right)\left[N_{1}, N_{1}\right]+N_{2}\left(N_{1}\left[N_{1}, N_{1}\right]\right) \subseteq N_{2} A=0 .
\end{gathered}
$$

Therefore,

$$
\left(N_{1}\left[N_{1}, N_{1}\right]\right)\left(N_{2}\left[N_{1}, N_{1}\right]\right) \subseteq\left(N_{1}\left(N_{2}\left[N_{1}, N_{1}\right]\right)\right)\left[N_{1}, N_{1}\right]=0 .
$$

Since $N_{2}\left[N_{1}, N_{1}\right] \subseteq N_{1}$ it follows that $\left(N_{2}\left[N_{1}, N_{1}\right]\right)^{2} \subseteq N_{1}\left(N_{2}\left[N_{1}, N_{1}\right]\right)=0$. Since $\left[N_{1}, N_{1}\right] \subseteq$ $N_{2}$, using Lemma 9 we get $\left[N_{1}, N_{1}\right]^{2} \subseteq\left[N_{1}, N_{1}\right] N_{2}=0$. Therefore, by Lemma 8 and Lemma 9, we also get

$$
K^{2} \subseteq\left(N_{1}\left[N_{1}, N_{1}\right]\right)\left(N_{2}\left[N_{1}, N_{1}\right]\right)+\left(N_{2}\left[N_{1}, N_{1}\right]\right)\left[N_{1}, N_{1}\right] \subseteq N_{1}\left[N_{1}, N_{1}\right] \subseteq N_{1} N_{2} \subseteq A .
$$

Then $K^{(2)}=0$.

Similarly, $L=N_{2}\left[N_{2}, N_{2}\right]+N_{1}\left[N_{2}, N_{2}\right]+\left[N_{2}, N_{2}\right]$ is a ideal of the algebra $N$ and $L^{(2)}=0$. Therefore, $K+L$ is a solvable ideal of the algebra $N$. From the solvability of the quotient algebra $N /(K+L)$ follows the solvability of $N$. We have $\left[\overline{N_{i}}, \overline{N_{i}}\right]=0$ in the quotient algebra $N /(K+L)$, where $\overline{N_{i}}$ is the image of $N_{i}$ in $N /(K+L), i=1,2$.

Hence we can assume that $\left[N_{1}, N_{1}\right]=\left[N_{2}, N_{2}\right]=0$ in the algebra $N$.

The proof of the Proposition 2. Let $x_{1}, y_{1} \in N_{1}, x_{2} \in N_{2}$. Then

$$
\begin{aligned}
& \left(x_{1} y_{1}\right) x_{2} \stackrel{\text { by }(2)}{=}\left(x_{1} x_{2}\right) y_{1} \stackrel{\text { by Lemma } 8}{=}-\left(x_{2} x_{1}\right) y_{1}=-\left(x_{2}, x_{1}, y_{1}\right)-x_{2}\left(x_{1} y_{1}\right) \stackrel{\text { by }(1) \text { and Lemma } 11}{=} \\
& -\left(x_{1}, x_{2}, y_{1}\right)-\left(x_{1} y_{1}\right) x_{2} \stackrel{\text { by Lemma }}{=}{ }_{12}^{10}-\left(x_{1} x_{2}\right) y_{1}-\left(x_{1} y_{1}\right) x_{2} \stackrel{\text { by }(2)}{=}-2\left(x_{1} y_{1}\right) x_{2} .
\end{aligned}
$$


Hence $3\left(x_{1} y_{1}\right) x_{2}=0$. Therefore, $N_{1}^{2} N_{2}=0$. Then $N_{2} N_{1}^{2}=0$ by Lemma 11. Similarly, $N_{2}^{2} N_{1}=0$ and $N_{1} N_{2}^{2}=0$

Consequently, $N_{1}^{2} N_{1}^{2} \subseteq N_{1}^{2} N_{2}=0$ and $N_{2}^{2} N_{2}^{2} \subseteq N_{2}^{2} N_{1}=0$. Moreover, $N_{1}^{2} N_{2}^{2} \subseteq$ $\left(N_{1} N_{2}^{2}\right) N_{1}=0$. Similarly, $N_{2}^{2} N_{1}^{2}=0$.

Let $I=N_{1} N_{2}+N_{1}^{2}+N_{2}^{2}$. By Lemma $1, I^{2} \subseteq N_{1}^{2}+N_{2}^{2}$ and $I^{(2)}=0$. By Corollary 3 and Lemma 8, we get $N^{(2)} \subseteq I$. Hence the algebra $N$ is solvable.

Theorem 2. Let $F$ be a field of characteristic $\neq 3$ and let $N=N_{0}+N_{1}+N_{2}$ be a $\mathbb{Z}_{3}$-graded Novikov algebra. Suppose that $N_{0}$ is a solvable algebra. Then $N$ is a solvable algebra.

Proof. Let $A=N_{0}$ be a solvable algebra with solvability length $n \geq 1$. If $n=1$, then $N$ is solvable by Proposition 2. Suppose that $n \geq 2$, that is $A^{2} \neq 0$. Let $I$ be the ideal of $N^{2}$ from Lemma 6. Recall that $N^{(2)} \subseteq I$. Therefore, it is sufficient to prove that $I$ is a solvable ideal of $N$.

Let $K$ be the ideal of $I$ from Lemma 7. Since $\left(A^{2}+N_{1} N_{2}+N_{2} N_{1}\right)^{2} \subseteq A^{2}$, the quotient algebra $I / K$ is again solvable by Proposition 2. Therefore, $I^{(s)} \subseteq K$ for some positive integers $s$.

Notice that the 0 -component of $K$ is $A^{2}$ and has the solvability length $n-1$. Leading an induction on $n$ we may assume that $K$ is solvable. Consequently, $I$ and $N$ are both solvable.

Corollary 4. Let $n$ be a positive integer of the form $n=2^{s} 3^{t}>1$ for some nonnegative integers $s, t$. Let $N$ be a $\mathbb{Z}_{n}$-graded Novikov algebra over a field of characteristic $\neq 2,3$. If $N_{0}$ is solvable, then $N$ is solvable.

This is a standard corollary of Theorems 1 and 2 (see, for example [28]).

The right powers of an arbitrary algebra $A$ are defined inductively by $A^{[1]}=A$ and $A^{[n]}=A^{[n-1]} A$ for all integers $n \geq 2$. An algebra $A$ is called right nilpotent if $A^{[n]}=0$ for some positive integer $n$. I. Shestakov and Z. Zhang recently proved [13] that every solvable Novikov algebra is right nilpotent.

Corollary 5. Let $n$ be a positive integer of the form $n=2^{s} 3^{t}>1$ for some nonnegative integers $s, t$. Let $N$ be a $\mathbb{Z}_{n}$-graded Novikov algebra over a field of characteristic $\neq 2,3$. If $N_{0}$ is right nilpotent, then $N$ is right nilpotent.

\section{Acknowledgments}

The first author is supported by the grant AP05133009 of MES RK. The second author is supported by the Program of fundamental scientific research of the SB of RAS, project 0314-2016-0001.

\section{REFERENCES}

[1] I.M. Gel'fand and L.A. Diki, "Fractional powers of operators and Hamiltonian systems", Funct. Anal. Appl., 1976, 10:4, 259-273. DOI: 10.1007/BF01076025.

[2] I.M. Gel'fand and I.Ya. Dorfman, "Hamiltonian operators and algebraic structures related to them", Funct. Anal. Appl., 1979 13:4, 248-262. DOI:10.1007/BF01078363. 
[3] B.A. Dubrovin, S.P. Novikov, "Hamiltonian formalism of one-dimensional systems of hydrodynamic type, and the Bogolyubov-Whitman averaging method" Sov. Math., Dokl., 1983, 27:4, 665-669.

[4] B.A. Dubrovin, S.P. Novikov, "On Poisson brackets of hydrodynamic type", Sov. Math., Dokl. 1984, 30, 651-654.

[5] A.A. Balinskii, S.P. Novikov, "Poisson brackets of hydrodynamic type, Frobenius algebras and Lie algebras", Sov. Math., Dokl., 1985, 32, 228-231.

[6] X. Xu, "Hamiltonian operators and associative algebras with a derivation", Lett. Math. Phys., 1995, 33:1, 1-6. DOI: 10.1007/BF00750806.

[7] X. Xu, "Hamiltonian superoperators", J. Phys. A, Math. Gen., 1995, 28:6, 1681-1698. DOI: 10.1088/0305-4470/28/6/021.

[8] J.M. Osborn, "Novikov algebras", Nova J. Algebra Geom. 1992, 1:1, 1-13.

[9] J.M. Osborn, "Simple Novikov algebras with an idempotent", Comm. Algebra, 1992, 20, 2729-2753. DOI: $10.1080 / 00927879208824486$.

[10] J.M. Osborn, "Infinite dimensional Novikov algebras of characteristic 0", J. Algebra, 1994, 167:1, 146-167. DOI: 10.1006/jabr.1994.1181.

[11] E.I. Zel'manov, "A class of local translation-invariant Lie algebras", (Russian) Dokl. Akad. Nauk SSSR 292 (1987), no. 6, 1294-1297.

[12] V.T. Filippov, "On right-symmetric and Novikov nil algebras of bounded index", (Russian) Mat. Zametki 70 (2001), no. 2, 289-295; translation in Math. Notes 70 (2001), no. 1-2, 258-263. DOI: 10.1023/A:1010215127478

[13] I. Shestakov, Z. Zhang, "Solvability and nilpotency of Novikov algebras", Comm. Algebra, 2020, 48, 5412-5420. DOI: 10.1080/00927872.2020.1789652.

[14] G. Higman, "Groups and rings which have automorphisms without non-trivial fixed elements", J. London Math. Soc., 1957, 32:2, 321-334. DOI: 10.1112/jlms/s1-32.3.321

[15] A.I. Kostrikin, V.A. Kreknin, "Lie algebras with a regular automorphism", Sov. Math. Dokl., 1963, 4, 355-358.

[16] V.A. Kreknin, "Solvability of a Lie algebra containing a regular automorphism", Sib. Math. J., 1967, $8: 3,715-716$.

[17] N.Yu. Makarenko, "A nilpotent ideal in the Lie rings with automorphism of prime order", Sib. Mat. Zh., 2005, 46:6, 1360-1373. DOI:10.1007/s11202-005-0104-0

[18] G.M. Bergman, I.M. Isaacs, "Rings with fixed-point-free group actions", Proc. London Math. Soc., 1973, 27, 69-87. DOI: 10.1112/plms/s3-27.1.69

[19] V.K. Kharchenko, "Galois extensions and quotient rings", Algebra and Logic, 1974, 13:4, $265-281$. DOI: $10.1007 /$ BF01463143.

[20] W.S. Martindale, S. Montgomery, "Fixed elements of Jordan automorphisms of associative rings", Pacific J. Math, 1977, 72:1, 181-196. DOI: 10.2140/pjm.1977.72.181.

[21] A.P. Semenov, "Subrings of invariants of a finite group of automorphisms of a Jordan ring", Sib. Math. J., 1991, 32:1, 169-172. DOI:10.1007/BF00970181.

[22] E.I. Zel'manov, "On solvability of Jordan nil-algebras", Sib. Adv. Math. 1991, 1, 185-203; translation from Tr. Inst. Mat., 1989, 16 , 37-54.

[23] V.N. Zhelyabin, "Jordan superalgebras with a solvable even part", Algebra and Logic, 1995, 34:1, 25-34. DOI:10.1007/BF00750553.

[24] O.N. Smirnov, "Solvability of alternative $\mathbb{Z}_{2}$-graded algebras and alternative superalgebras", Sib. Math. J., 1991, 32:6, 1030-1034. DOI:10.1007/BF00971210.

[25] K.A. Zhevlakov, "Solvability of alternative nil-rings", Sib. Math. J., 1962, 3, 368-377.

[26] G.V. Dorofeev, "An instance of a solvable, though nonnilpotent, alternative ring", Uspehi Mat. Nauk, 1960, 15:3, 147-150. DOI: 10.1090/trans2/037/08.

[27] K.A. Zhevlakov, A.M. Slinko, I.P. Shestakov, A.I. Shirshov, "Rings that are Nearly Associative", translated from the Russian by Harry F. Smith, Academic Press, New York, 1982.

[28] M. Goncharov, "On solvable $\mathbb{Z}_{3}$-graded alternative algebras", Algebra and Discrete Mathematics, 2015, 20:2, 203-216. 